\title{
THE MD PhD PHYSICIAN SCIENTIST-ENDANGERED SPECIES OR THE NEXT GENERATION?
}

\author{
Gary J. Nabel, M.D., Ph.D. \\ Howard Hughes Medical Institute, University of Michigan Medical \\ School, Ann Arbor, Michigan, U.S.A.
}

The success of biomedical research in recent decades has provided unique opportunities to advance the practice of modern medicine. At the same time, enormous challenges face physicians in their efforts to use expanding knowledge to treat disease. There has been considerable progress in molecular medicine, but an argument can still be made that the advances have not had a significant impact on the incidence and prognosis of common diseases, including cardiovascular disease, cancer, and acquired immunodeficiency syndrome (AIDS). The complexity of human pathophysiology, together with the technical sophistication in the practice of medicine, has made it difficult to bridge disciplines which must productively interact to progress. Among those who could potentially synthesize this information is the dual degree physician scientist, the MD PhD. Yet as the need for such individuals increases, so also do the pressures which make it difficult for them to succeed. Students must master an increasing knowledge of basic science, face uncertainty about their ultimate role in academic medicine, and adjust to substantial economic pressures in both academic medicine and clinical practice. The question is: Do MD PhD scientists represent the great hope for the future of medicine, or are they an endangered species?

Medical science has always balanced two competing philosophies. Different approaches lead to success in the clinic compared with the lab. On the one hand, physicians must make decisions about patients using available information which is often limited. Medical school teaches students to find expedient solutions to problems with incomplete knowledge. In contrast, researchers demand precise and rigorous answers. Research training focuses on a careful understanding of selected problems, often simplified to limit variables, and rigorous experiments are designed to maximize information in a stepwise logical fashion. Each discipline can be all consuming; obtaining either an $\mathrm{MD}$ or $\mathrm{PhD}$ degree alone is a long and arduous process, requiring complete focus and commitment.

A relatively small number of individuals obtain the combined MD PhD degree. Programs often extend to 7 or 8 years. This long training period may argue for separation of degrees; by the time two degrees are obtained and postgraduate training completed, the period of peak scientific productivity may have begun to pass. Also, combined training does not change the ultimate outcome. Realistically, students are required to choose between performing research or practicing a clinical specialty. It is difficult to spend 4 to 5 years of advanced clinical training and then decide to go back to the lab without continued laboratory exposure.

As an MD PhD graduate, my own realization of this fact came as a 3rd year resident at the Brigham and Women's Hospital in Boston, Massachusetts, U.S.A., when I was arranging for a postdoctoral fellowship. Having visited David Baltimore's lab, where I eventually pursued my studies, I began to attend lab meetings while still on service in the hospital. The dichotomy between the two worlds became quickly evident. At Brigham and Women's Hospital, I was working in the intensive care unit, rounding on extremely sick, complicated patients. Examining the nuances of their management were exceptionally sophisticated physicians. They had acquired a wealth of experience, an excellent basic science background, and were well read in the clinical literature. Arriving at the Massachusetts 
Institute of Technology-just a drive over the bridge to Cambridge - to attend a lab meeting, I was suddenly immersed in an entirely different culture, surrounded by postdoctoral fellows who were equally bright, high powered, rigorous, and well read, but entirely dedicated to research. Each group demonstrated sophistication, specialization, and complete commitment. This juxtaposition within the space of one hour reinforced the notion that, as an MD PhD, I would not be able to do half-time what both groups were pursuing full-time. I had to make a choice.

Nonetheless, a combined program does provide valuable training. Medical school training grants an appreciation for the pathophysiology and consequences of human diseases; students have the privilege of caring for patients and their families. From the laboratory comes the rigor to pursue basic science, an understanding of the effort required to make progress in studying a problem, and the risks and rewards of addressing exciting new scientific areas. Together, these experiences give the $\mathrm{MD} \mathrm{PhD}$ student a unique appreciation of the potential for both disciplines. Training as a physician who cares for a patient, who has an interest in treating the problems, and as a scientist concerned primarily with understanding them, provides greater insight into molecular medicine and the background to design studies that maximize an understanding of interventions or disease processes, and ultimately optimize the likelihood of benefiting patients.

This notion was reinforced when gene transfer studies in humans began in my laboratory. For a long time, such protocols were largely an academic exercise. To justify human studies, it was necessary to understand mechanisms, justify hypotheses, prove feasibility, and be convinced that the approach and the treatment rationale were logical. As soon as approval was received and brought to the patient, the agenda changed. For the patient, the underlying scientific rationale was of interest, but of secondary importance. The patient simply wanted to know one thing: Was this treatment going to help? Though a particular treatment may indeed not help the patient at hand, the hope is that this attempt to understand the disease better will help future patients.

In the end, the decision to pursue a combined training program must include some consideration of the cost versus the benefit, for both the individual and society. The MD PhD student makes a commitment to a prolonged training period, and often personal sacrifices must be made. In return, there are rich educational opportunities. For society, the cost of having to support the additional years of training for these students is offset by the benefit that comes from physician scientists who are committed to the basic science of human disease, who may help improve methods of diagnosis, treatment, and prophylaxis.

Knowledge of human biology is increasing at an exponential rate, but there is no guarantee that it will be rapidly and intelligently applied to clinical medicine. Reforms in health care are likely to lead to increased fragmentation of basic science and clinical medicine. Individuals with an understanding of both disciplines are poised to synthesize information and to advance molecular medicine. Dual degree training fulfills an important need and should be encouraged, for as time passes there will be more opportunities than ever to make use of this training. 